\title{
Special issue on advances in multimedia interaction and visualization
}

\author{
Rita Francese ${ }^{1} \cdot$ Ebad Banissi $^{2} \cdot$ Nuno Datia $^{3} \cdot$ Michele Risi $^{1}$ \\ Published online: 4 February 2022 \\ (C) The Author(s), under exclusive licence to Springer Science+Business Media, LLC, part of Springer Nature 2022
}

Information Visualization is the field of study concerned with the development of methods for transforming abstract, complex data into visual representations in order to make that data more easily communicable and understandable. Technological advances enable information visualization in many areas, such as Visual Analytics, social media, geo-sociology, health, business, security and more.

Interactivity and visualization increase the engagement with users, as they place them at the core of processes of access, dialogue, and relation with data providing visualization models for easy-to-use interactive systems. The use of interactive visualization in multimedia provides a powerful means for investigating, exploring, and imagining new communication modalities we will use in the near future.

This special issue collected emerging contributions in the area of multimedia interaction and its applications for information visualization with different types of data. It includes a selection of the best papers presented at iV 2019, the 23rd International Conference on Information Visualizations. The conference took place in two different locations: 02-05 July 2019 in Paris (France) and 16-19 July 2019 in Adelaide, Australia. Participants included $70+$ researchers from more than 23 countries.

Eleven papers have been accepted for this special issue by considering the technical quality, the originality, and the innovation of the proposed ideas, solutions, and systems: the authors

Rita Francese

francese@unisa.it

Ebad Banissi

banisse@1sbu.ac.uk

Nuno Datia

datia@isel.ipl.pt

Michele Risi

mrisi@unisa.it

1 Department of Informatics, University of Salerno, Fisciano, Italy

2 London South Bank University, London, UK

3 Instituto Superior de Engenharia de Lisboa (ISEL) \& NovaLINCS, Lisbon, Portugal 
introduced visualization and interaction approaches with various types of data appliable to different domains, ranging from communications, diagnostics, decision support, education, and gaming.

Papers $[4,6]$ investigated research aspects of the cinematographic field. In particular, the former addresses the problem of designing a visualization approach suitable for viewing and browsing a large collection of movies, while the latter proposes a system for the production of web-based interactive fictions.

Biomedical data have been analyzed and visualized by Frasca \& Tortora [5] to identify correlations between the medical reports of Parkinson's patients. To correlate the information of each patient's medical report, Information Retrieval and Machine Learning techniques have been adopted, including the Latent Semantic Analysis, Text2Vec, and Doc2Vec techniques. The authors proposed to visualize the correlations among medical reports with an interactive chart by using the D3 framework.

Educational research aspects have been addressed in [10,11] in Mathematics and Engineering education, respectively. To motivate children in learning the multiplication tables Rebotto et al. [10] proposed an Augmented Reality gamification approach based on two games, one turn-based fighting and other throwing-objects games. Computational fluid dynamics simulations is largely adopted in engineering education, broad engineering and business. Solmaz and Gerven [11] presented a system for generating dedicated workflows for computational fluid dynamics simulations in any kind of AR/VR environment.

In [7,9] the coupling of Artificial Intelligence with visualization and interaction has been investigated for enhancing decision support. Situated visualization in AR modality has been proposed in [7] to obtain more effective and efficient decision-making. Data representations are displayed near the data referent, providing the user with contextual, relevant, and appropriate information. Nazemi et al. concentrated their attention on strategic decision making which can be enhanced through awareness of emerging trends and may improve the firm's competitiveness and market position. Their work introduces a novel interaction design by investigating the main ideas from technology and innovation management and detection. Architecture and product design support has been addressed in [2], where an interactive visualization system supporting team members in the understanding how to assemble a product is proposed. The problem of a multiuser visualization of data on a mobile device has been addressed by Miranda et al. [8]. They presented a hybrid proposal of an augmented reality prototype to interacting with cards through the mobile device to support the process of creating data visualization. An AR environment enables multiple users to interact simultaneously.

Specific visualizations problems have been investigated in [1,3]. Chen and CUI addressed the problem of feature points missing and surface holes after 3D reconstruction, while the problem of visualizing data in high-dimensional space by exploiting machine learning dimensionality reduction techniques has been studied by Behera et al. [1].

\section{References}

1. Behera AP, Singh J, Verma S, Kumar M (2022) Data visualization through non linear dimensionality reduction using feature based Ricci flow embedding. Multimed Tools Appl 3:1-20 
2. Chang TW, Chen CY, Huang HY, Hsieh TL, Huang W, Datta S (n.d.) ViDA: Developing a visualization system for a Design-Fabrication-Assembly (D-F-A) process

3. Chen H, Cui W (2021) Holes filling of scattered point cloud based on simplification. Multimed Tools Appl 20:1-21

4. Cohen-Kalaf M, Lanir J, Bak P, Mokryn O (2021) Movie emotion map: an interactive tool for exploring movies according to their emotional signature. Multimed Tools Appl 12:1-22

5. Frasca M, Tortora G (2021) Visualizing correlations among Parkinson biomedical data through information retrieval and machine learning techniques. Multimed Tools Appl 27:1-9

6. Gómez-Albarrán M, Sarasa-Cabezuelo A, Sierra-Rodríguez JL, Temprado-Battad B (2021) Authoring and playing interactive fiction with conventional web technologies. Multimed Tools Appl 19:1-43

7. Martins NC, Marques B, Alves J, Araújo T, Dias P, Santos BS (2021) Augmented reality situated visualization in decision-making. Multimed Tools Appl 5:1-24

8. Miranda BP, Queiroz VF, Araújo TD, Santos CG, Meiguins BS (2021) A low-cost multi-user augmented reality application for data visualization. Multimed Tools Appl 23:1-29

9. Nazemi K, Burkhardt D, Kock A (2021) Visual analytics for technology and innovation management. Multimed Tools Appl 20:1-28

10. Rebollo C, Remolar I, Rossano V, Lanzilotti R (2021) Multimedia augmented reality game for learning math. Multimed Tools Appl 31:1-8

11. Solmaz S, Van Gerven T (2021) Automated integration of extract-based CFD results with $A R / V R$ in engineering education for practitioners. Multimed Tools Appl 4:1-23

Publisher's note Springer Nature remains neutral with regard to jurisdictional claims in published maps and institutional affiliations. 